\title{
Hybrid Elephant Herding and Particle Swarm Optimizations for Optimal DG Integration in Distribution Networks
}

\author{
Pushpendra Singh ${ }^{1,2}$, Nand K. Meena ${ }^{3, *}$, Shree Krishna Bishnoi ${ }^{4}$, Balvinder Singh ${ }^{2}$, and \\ Mahendra Bhadu ${ }^{4}$ \\ ${ }^{1}$ Department of Electrical Engineering, Rajasthan Technical University, Kota, India 302017 \\ ${ }^{2}$ Department of Electrical Engineering,Govt. Women Engineering College, Ajmer, India \\ ${ }^{3}$ School of Engineering \& Applied Science, Aston University, Birmingham B4 7ET, United Kingdom \\ ${ }^{4}$ Department of Electronics and Communication Engineering, Govt. Engineering College, Bikaner, India \\ ${ }^{5}$ Department of Electrical Engineering, Govt. Engineering College, Bikaner, India
}

\begin{abstract}
In this article, the amalgamation of two well-established meta-heuristic optimization methods is presented to solve the multi-objective distributed generation (DG) allocation problem of distribution systems. To overcome some of the shortcomings of newly developed elephant herding optimization (EHO), an improvement is suggested and then, a prominent feature of particle swarm optimization is introduced to the modified version of EHO. The suggested modifications are validated by solving a single objective DG integration problem where various performance parameters of the proposed hybrid method are compared with their individual standard variants. After validation, the proposed technique is exploited to solve a multi-objective DG allocation problem of distribution systems, aiming to minimize power loss and node voltage deviation while simultaneously maximizing the voltage stability index of three benchmark distribution systems namely, 33-bus, 69-bus and 118-bus. The obtained simulation results are further compared with that of the same available in the existing literature. This comparison reveals that the proposed hybrid approach is promising to solve the multi-objective $D G$ integration problem of distribution systems as compared to many existing methods.
\end{abstract}

\section{Nomenclature}

$N$

$\mathrm{L}_{\text {center }, n}$

$P_{j}, P_{k}$

$L_{\text {worst }, n}$

$Q_{j}, Q_{k}$

$\beta$

$r_{j k}$

$\alpha$

$V_{j}, V_{k}$

$\operatorname{rand}(1)$

$\delta_{j}, \delta_{k}$

$n_{z}$
Total number of buses in the system.

Mean position of elephant in $n$th clan.

Real power injections at buses $j$ and $k(\mathrm{~kW})$.

The position of worst bull in $n$th clan.

Reactive power injections buses $j$ and $k$ (kVAr).

Scale factor between 0 to 1 .

Resistance of line connecting buses $j$ and $k(\Omega)$.

A scale factor varying between 0 and 1 .

Magnitude of voltage at bus $j$ and $k$ (p.u.).

Random number between 0 and 1 .

The voltage angles at $j$ th and $k$ th buses (radian).

Total number of elephants 


\begin{tabular}{|c|c|}
\hline$x_{j k}$ & Reactance of branch between node $j \& k(\Omega)$. \\
\hline$L_{\max }, L_{\min }$ & Max and min limits of clan. \\
\hline$S_{D G}^{M a x}$ & Maximum DG capacity allowed on a bus (kVA). \\
\hline$v_{i}^{k}$ & Velocity of elephant $i$ in iteration $k$. \\
\hline$S_{D G_{j}}$ & Suggested capacity of DG at bus $j(\mathrm{kVA})$. \\
\hline$s_{i}^{k}$ & Position of particle $i$ in iteration $k$. \\
\hline$Y_{j k}$ & Element of Y-bus matrix $\left(\Omega^{-1}\right)$ \\
\hline$c_{1}, c_{2}$ & The acceleration coefficients. \\
\hline$I_{j k}, I_{j k}^{\max }$ & $\begin{array}{l}\text { Instantaneous and maximum allowed currents of lines connecting nodes } j \\
\text { and } k \text { (Amp). }\end{array}$ \\
\hline pbest $_{i}$ & Best location of particle based on its own experience. \\
\hline$\phi_{j k}$ & Impedance angle of node $j$ and $k$ (radian). \\
\hline gbest $_{i}$ & The best location of particle based on overall swarm experience. \\
\hline$\sigma_{j}$ & Binary decision variable. \\
\hline$S^{P e a k}$ & Peak demand of the system (kVA). \\
\hline$\delta_{t}$ & The time step \\
\hline$w$ & The inertial weight taken. \\
\hline$L_{n e w, n, i}$ & New position of $i$ th elephant of $n$th clan. \\
\hline$w(k)$ & Inertial weight of particle in iteration $k$. \\
\hline$L_{n, i}$ & Current position of elephant $i$ in clan $n$. \\
\hline$w_{\min }$ & Minimum inertial weight of final value. \\
\hline$L_{b e s t, n}$ & The matriarch position in $n$th clan. \\
\hline$w_{\max }$ & The maximum inertia weight value. \\
\hline$L_{b e s t, n, i}$ & Best position of elephant $i$ in clan $n$. \\
\hline$i t e r_{\max }$ & Maximum number of iterations. \\
\hline$k_{1}, k_{2}$ & Weighing coefficients. \\
\hline
\end{tabular}

\section{Introduction}

Distributed generation (DG) has provided new dimensions to the electric power industry. Usually, the generation capacity of DGs varies from fewer kilowatts to some megawatts and placed in the vicinity of the load [1]. Nowadays, different types of DG technologies are available and each DG technology has a different profound impact on system performance and economics [2]. The increasing concern of eco-friendly power generation has motivated power system planners to increase the share of renewables. It promotes DG integrations in small to large-scale power distribution networks across the globe. The optimal integration of DGs enhances the reliability, quality and performance of 
power networks. Whereas, a non-optimal allocation of DGs may provide counterproductive results [3]. Generally, the optimally placed DGs can provide, reduction in power [4-8] and energy losses [9], peak load shaving [1], improvement in bus voltage profile [3-6,9], enhanced stability [3-6], improved power quality and reliability.

In practice, the utilities are trying to maximize various techno-economic and social benefits simultaneously by satisfying enormous constraints. Therefore, it is advantageous to formulate the DG integration models as a multi-objective optimization problem. In recent years, various single $[7,8]$ and multi-objective [3-6] DGs allocation problems have been solved for distribution systems. The optimal allocation of DGs in distribution networks is generally formulated as a mixed-integer, non-convex, and non-linear optimization problem by considering type, number, location and size of DGs [3,6]. The determination of optimal sites and sizes of DGs, out of enormous feasible solutions, is a challenging task, therefore, it requires powerful optimization techniques to obtain the global optimal solution. In literature, various optimization methods have been suggested to solve the DG allocation problems of distribution systems namely, analytical, statistical, numerical, artificial intelligence (AI), and hybrid optimization techniques. The conventional or analytical methods may include $2 / 3$ rule and analytical methods, [10, 11]. The conventional approaches are based on some set of rules, linearization, and simplified assumptions, therefore, have some shortcomings such as lack of robustness, limitation towards single-objective optimization problems of small to medium-sized distribution systems $[2,12]$. Similarly, numerical methods are also found to be very efficient and fast however subjected to the accurate modeling of the problem [13]. Nowadays, AI-techniques are found to be very effective to solve complex real-life engineering optimization problems. The nature-inspired techniques play a vital role to solve complex and non-linear DG allocation problems [14].

The popularly used nature-inspired/AI optimization techniques are genetic algorithm (GA) [5,15], particle swarm optimization (PSO) [5,15,16], harmony search algorithm (HSA) [17], teaching learning-based optimization (TLBO) $[4,18]$, simulated annealing (SA) [7], invasive weed optimization (IWO) [8], elephant herding optimization (EHO) [6], water cycle algorithm, [19], salp swarm algorithm [20], lightning search algorithm [14], etc. It has been observed that AI techniques are able to search the global optima for complex real-life engineering optimization problems although these are computationally demanding. Moreover, some of the standard variants of these optimization methods show some limitations when applied to complicated practical engineering optimization problems. For example, GA has a tendency of premature convergence and local trapping, whereas PSO and SA show relatively poor global searching performance [12].

In recent, many improved optimization methods have been developed to overcome some of the limitations identified in their standard variants. The popularly known improved optimization techniques may include quasi-oppositional TLBO (QOTLBO) [4], intelligent search-based TLBO [21], improved elephant herding optimization (IEHO) [6], improved PSO [9], improved multi-objective harmony search (IMOHS), [22], improved shuffled frog leaping algorithm [23], dynamic node priority-based GA [24], modified moth search optimization [25], etc. Besides, some hybrid optimization methods are also suggested by considering the propitious traits of their standard variants. In $[5,15]$, a hybrid GA/PSO method is proposed to overcome some limitations of GA and PSO. A loss sensitivity factor-based SA (LSFSA) is proposed in [7] to solve the DG allocation problem of distribution systems. In [26], an effective hybrid 
PSOGSA approach is proposed by combining particle swarm and gravitational search optimization techniques for optimal integration of renewable-based DGs. In 2015, Wang et al. [27], developed a new nature-inspired, populationbased, optimization technique inspired by herding behaviour of elephants, i.e., elephant herding optimization (EHO). The method has shown some promising capabilities while solving various benchmark functions. However, this method has some limitations when applied to real-life engineering optimization problems, as identified by [6].

In this article, an effective and hybrid optimization method is developed to solve complex real-life DG allocation problems of distribution networks. The proposed approach effectively introduced promising traits of well-known PSO technique to recently developed EHO method to overcome some of the limitations of standard EHO [27]. The insertion of traits is done in such a way that the basic mechanism of EHO remains unaltered. Two major corrections are suggested to update the positions of best and worst elephants of the herd. The suggested improvements are demonstrated by solving a single objective DG integration problem of 33-bus distribution system and then its performance is compared with standard and improved variants of EHO and PSO. Furthermore, the hybrid EHOPSO is applied to solve a multi-objective DG allocation problem of three standard distribution systems of 33, 69 buses and 118 buses. The obtained simulation results are further compared with some of the existing optimization methods adopted to solve the DG integration problems. The comparisons reveal that the proposed approach is providing promising results.

\section{Problem Formulation}

In practice, the distribution companies have to achieve multiple objectives simultaneously, such as minimization of power loss, voltage regulation, power quality and reliability. This section covers the mathematical modeling of the proposed multi-objective DG integration problem of distribution system focusing to optimize multiple objectives of equal importance simultaneously. In the proposed DG allocation problem, three objectives have been considered namely system power loss minimization, node voltage deviation minimization and voltage stability index maximization, as discussed in the following sections.

\subsection{Minimization of system power loss $\left(f_{1}\right)$}

The minimization of system power loss may be considered as one of the vital objectives since it affects annual revenue of distribution network operator (DNO). Therefore, it is considered as one of the objective function of DG integration in distribution systems. The system power loss is expressed as

$$
\min f_{1}=\sum_{j=1}^{N} \sum_{k=1}^{N} \frac{r_{j k}}{V_{j} V_{k}} \cos \left(\delta_{j}-\delta_{k}\right)\left(Q_{j} Q_{k}+P_{k} P_{j}\right)+\frac{r_{j k}}{V_{j} V_{k}} \sin \left(\delta_{j}-\delta_{k}\right)\left(Q_{j} P_{k}-Q_{k} P_{j}\right)
$$

\subsection{Minimization of node voltage deviation $\left(f_{2}\right)$}

Node voltage deviation is one of the popular indicators of system voltage quality. The modern power consumers are much more concerned about voltage quality therefore, voltage regulation turns out to be one of the important 
objectives of distribution utilities. In the proposed DG allocation problem, the minimization of node voltage deviation is also considered as one of the objectives, as expressed below.

$$
\min f_{2}=\sum_{j=1}^{N}\left[V_{j}-V_{\text {rated }}\right]^{2}
$$

\subsection{Improvement of voltage stability index $\left(f_{3}\right)$}

The improvement of node voltage profile has not been a sufficient criterion to show the quality of voltage. To maintain the node voltages within the prescribed limits over variable loading, voltage stability index (VSI) has been suggested in [28] which is further investigated in [3-6]. The utilities always try to maintain VSI values of all branches of the network close to unity, as it secures the system. To improve the voltage stability of complete system, a branch with minimum VSI value is selected and its VSI value is maximized. The objective of voltage stability improvement is expressed as

$$
\min f_{3}=\frac{1}{\min \left\langle V S I_{j k}\right\rangle} \quad \forall j, k
$$

where,

$$
V S I_{j k}=V_{k}^{4}-4\left(P_{j} r_{j k}+Q_{j} x_{j k}\right) V_{k}^{2}-4\left(P_{j} x_{j k}-Q_{j} X_{j k}\right)^{2} \quad \forall j
$$

\subsection{Objective function}

A weighted-sum approach is adopted to solve the multi-objective DG integration problem of distribution systems. Mathematically, the following objective function has been formulated to optimally determine the optimal sites and sizes of DGs in distribution systems. The value of weights are referred from $[4,5]$.

$$
\min F=f_{1}+k_{1} f_{2}+k_{2} f_{3}
$$

subjected to following constraints:

Real power balance at the node,

$$
P_{j}=V_{j} \sum_{k=1}^{N} V_{k} Y_{j k} \cos \left(\phi_{j k}+\delta_{k}-\delta_{j}\right) \quad \forall j
$$

Reactive power balance at the node,

$$
Q_{j}=-V_{j} \sum_{k=1}^{N} V_{k} Y_{j k} \sin \left(\phi_{j k}+\delta_{k}-\delta_{j}\right) \quad \forall j
$$

Slack bus voltage and angle (per unit and radian),

$$
V_{1}=1.0 \text { and } \delta_{1}=0.0
$$

Voltage limits at the other buses (per unit),

$$
0.95 \leq V_{j} \leq 1.05 \quad \forall j
$$


Feeder capacity limits,

$$
0 \leq I_{j k} \leq I_{j k}^{M a x} \quad \forall j, k
$$

Maximum penetration limits on each bus,

$$
0 \leq S_{D G_{j}} \leq S_{D G}^{M a x} \quad \forall j
$$

Maximum DG penetration limits of the system,

$$
0 \leq \sum_{j=1}^{N} \sigma_{j} S_{D G_{j}} \leq S^{\text {Peak }} \quad \forall j
$$

\section{Elephant Herding optimization (EHO)}

EHO is a swarm-based, meta-heuristic optimization method proposed by Gai-Ge Wang et al. in 2015 [27]. It is inspired by the herding behavior of elephants. The elephant is a social animal and always like to live in herds, which mostly consist of female elephants (cows) and their children (calf/calves). Each clan has a leader known as matriarch elephant and clans of herd move under the guidance of their respective matriarch, as shown in Figure 1. Some studies on elephants [6] have revealed that cows secure their calf/calves from hurdles, starving predators, and find a safe place by communicating with other elephants by using foot stepping, stomping, ear flapping, etc. Generally, they produce seismic waves that can travel up to 30 kilometers along the surface. Sometimes, they also produce low frequency rambling. The research has revealed that the herd of elephants senses these vibrations and consider it a warning or danger signal. The same communication is also used to search for secure, safe, and comfortable shelters. In [27], this herding behavior is modeled into some set of mathematical equations and developed EHO method, presented in the following sections.

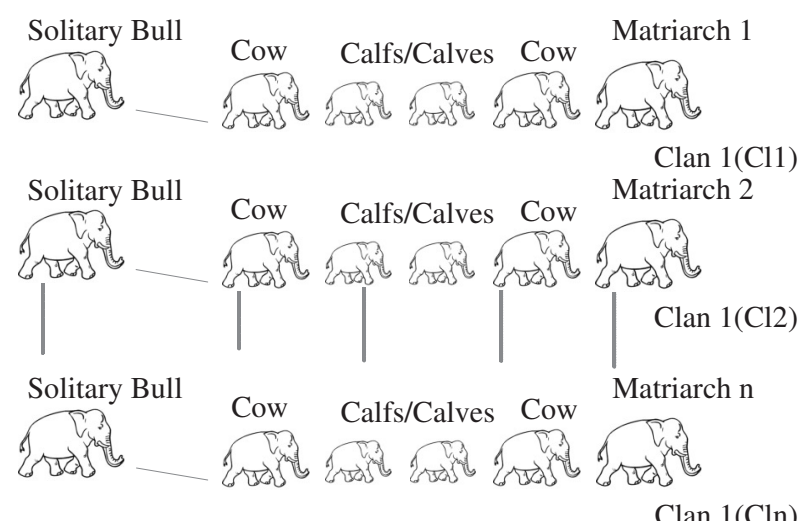

Figure 1. Social behavior of elephants adopted in EHO 


\subsection{Basic EHO}

In basic EHO, the following assumptions have been considered [27]: a) the herd consists of clans should have a fixed number of elephants; b) number of bulls separated from the herd should also be the same for all herds which will be living solitary but remain in contact with their family and; c) elephants of a clan live together under the leadership of matriarch. The essential steps of the method is describe below.

Step-1: In this step, the positions of elephants are updated in each clan, except best and worst elephants, as expressed below.

$$
L_{n e w, n, i}=L_{n, i}+\alpha\left(L_{b e s t, n}-L_{n, i}\right) \cdot \operatorname{rand}(1)
$$

Step-2: Now, the position of best elephant or matriarch is updated by following the mean position of the clans as given below.

$$
\begin{gathered}
L_{\text {new }, n, i}=\beta \times L_{\text {center }, n} \\
L_{\text {center }, n}=\frac{\sum_{i=1}^{n} L_{n, i}}{n_{z}}
\end{gathered}
$$

Step-3: As discussed, the bull will be separated from its clan as it grown-up. Therefore, considering this behavior, following separating operator is suggested to update the position of worst elephant.

$$
L_{w o r s t, n}=L_{\min }+\left(L_{\max }-L_{\min }+1\right) \cdot \operatorname{rand}(1)
$$

Step-4: Steps 1 to 3 are repeated till algorithm reaches to stopping criteria or maximum number of iterations.

\section{Particle Swarm Optimization}

The PSO algorithm is an evolutionary computational technique which is also inspired by social behavior of bird flocking, fish schooling and was developed by Eberhart and Kennedy in 1995 [16]. It is easy to implement therefore has received attention of researchers from diversified areas. The basic steps of PSO are described below.

Step-1 (Initialization): Initialize random but feasible population.

Step-2 (Fitness calculation): Calculate the fitness values of the individuals initialized in step 1 and obtain pbest and gbest.

Step-3 (Calculation of weight coefficient): Set values of weights $w_{\min }, w_{\max }$ and acceleration constants $c_{1}, c_{2}$, and then calculate the values of weight coefficients as

$$
w(k)=w_{\max }-\left(\frac{w_{\max }-w_{\min }}{\text { iter }_{\max }}\right) \cdot \text { iter }_{k}
$$

Step-4 (Update the velocity of particle): Update the velocity of particle by using following equation

$$
v_{i}^{k+1}=w \cdot v_{i}^{k}+c_{1} \cdot \operatorname{rand}(1) \cdot\left(\frac{\text { pbest }_{i}-s_{i}^{k}}{\Delta t}\right)+c_{2} \cdot \operatorname{rand}(1) \cdot\left(\frac{g b e s t_{i}-s_{i}^{k}}{\Delta t}\right)
$$

Step-5 (Update the position of particle): Update the position of particle as follows

$$
s_{i}^{k+1}=s_{i}^{k}+v_{i}^{k+1} \cdot \Delta t
$$


Step-6 (Correction): Apply a correction algorithm to correct infeasible individuals, if any.

Step-7 (Update best particle): Update the value of gbest, if better solution is obtain.

Step- $\boldsymbol{8}$ (Stopping criteria): Repeat steps 2 to 7 till convergence criteria or maximum iteration is achieved.

\section{Proposed Amalgamation of EHO and PSO}

This section covers the proposed hybrid EHO-PSO method that effectively hybridizes two well-known, nature-inspired optimization techniques namely, EHO and PSO. The standard variants of EHO and PSO have some similar inherent properties. For example, participating individuals are inspired by the position of other individuals and keep communicating with each other. However the standard variants of these algorithms have some inherent limitations such as PSO is suffering from poor convergence, [9]. The PSO method is not considering the position of worst particle while updating the position of other particles which results in the poor mean fitness. Similarly, the standard variant of EHO has some serious inerrant drawbacks while solving complex real-life optimization problems, as also identified in [6]. It may also be observed that the position of the best elephant is updated by following the mean behavior of clan which fluctuates when applied to complex real-life engineering optimization problems. The possible reason for this may be the randomness of the separating operator. Therefore, by introducing powerful attributes of PSO to EHO, some of the limitations of EHO may be removed. The existing limitations of EHO along with the suggested improvements are listed below.

Observation-I: As suggested in [27], the leader or matriarch elephant updates its position by receiving the mean information from all elephants in the clan so that the leader elephant can maintain its required distance by communicating to all clan elephants. The mean position of each clan is calculated in order to update the position of the best elephant as expressed in (14). However, the expression provides acceptable performance for benchmark functions but shows poor convergence for real-life engineering optimization problems with enormous constraints. This may happen due to the consideration of weak elephants in the mean calculation that may slow down clan and leader, results in the local convergence.

Suggested Correction-I: In order to overcome the observed limitation, an alternative mean i.e. mode is suggested to update the position of the best elephant. The mode calculation considers maximum elephants with common fitness values which helps the leader to judge the location of majority elephants in their respective clans thereby improves the performance of the algorithm. In this way, the number of best repetitive solutions will increase on the verge of convergence. Therefore, the position of the matriarch is now updated as follows.

$$
L_{n e w, n, i}=\beta \times L_{\text {center }, n}
$$

where,

$$
L_{\text {center }, n}=M o\left\langle L_{n, i}\right\rangle \quad \forall i
$$


Observation-II: In standard EHO, a separating operator has been suggested in Equation (16) to separate male elephants as they grow up. However, they remain in touch with their family groups with low-frequency vibrations. The separating operator is essential to improve the searching ability of the EHO method [27] as it uses stochastic and uniform distribution in a range of [0, 1]. In [27], positions of worst elephants are updated randomly which may deceive the algorithm and results in poor solutions. Furthermore, the standard variant of EHO does not consider the speed or velocity of elephants herd, which is also a deciding factor to search the global solution.

Suggested Correction-II: To overcome the above-stated limitation of basic EHO, a new velocity factor is introduced for worst elephants. The velocity component is used to update the position of the worst elephants so that they can maintain the required distance from their family groups. The longer distance between the worst elephant and family group can deteriorate communication instructions to be exchanged. In proposed work, the position of the worst elephant is updated as

$$
\begin{gathered}
L_{\text {worst }, n}=L_{n, i}+v_{\text {worst }, n, i} \times \Delta t \\
v_{\text {worst }, n, i}=w \times v_{n, i}+c_{1} \times \operatorname{rand}(1) \times \frac{L_{b e s t, n, i}-L_{n, i}}{\Delta t}+c_{2} \times \operatorname{rand}(1) \times \frac{L_{b e s t, n}-L_{n, i}}{\Delta t}
\end{gathered}
$$

The suggested correction-II would help the worst elephants to remain in touch with their family groups by maintaining their velocities and positions. Equation (23) is introduced from PSO by considering the positions of local and global best individuals in EHO. It will provide the direction signals and speed to the worst elephants towards their respective matriarch.

\section{Optimal Allocation of DGs using Proposed EHO-PSO Method}

In this section, the proposed hybrid EHO-PSO technique is adopted to solve the optimal DG integration problem of distribution systems. The structure of an individual used in the proposed algorithm includes information about the DGs' locations and sizes as expressed in Figure 2. As observed from the figure, the structure of an individual can

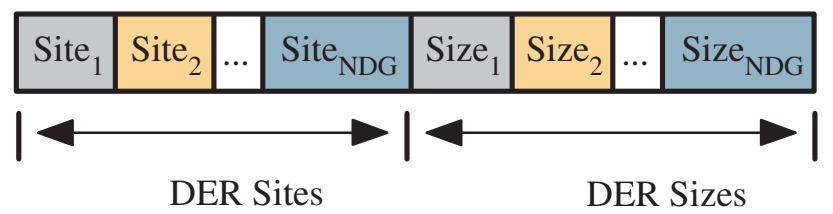

Figure 2. The structure of an individual used in proposed EHO-PSO for DG integration

be bifurcated in which the first part is comprised of DG locations. The information of respective DG capacities is given in the second half. The length of this individual depends on the number of DGs to be installed in a system. The basic steps of the proposed algorithm to optimally allocate DGs in distribution systems are presented below, followed by its flowchart in Figure 3.

Step-1: Initialize random population of elephants herd ' $h$ '. 
Step-2: Calculate the fitness of each individual using load flow calculations.

Step-3: Identify the matriarch (best elephant) and solitary bull (Worst elephant), based on fitness values.

Step-4: Update the positions of all elephants in each clan by using Equation (13), except the best and worst elephants.

Step-5: Update the positions of best and worst elephants, as suggested improvements in Equation (20) and Equation (22) respectively.

Step-6: Apply a correction algorithm to correct the infeasible individuals, if any.

Step-7: Repeat steps 2 to 6 till maximum number of iterations.

\section{Case Study and Discussions}

In this section, the proposed hybrid optimization method is applied to solve the multi-objective DG integration problems of three benchmark distribution systems namely 33, 69 buses and 118 buses. In order to validate the suggested corrections in EHO, a single objective DGs allocation problem is solved for 33-bus test system. Later, it is applied to solve the multi-objective DG allocation problem of distribution systems, presented in following sections.

\section{$7.1 \quad$ Validation of proposed method}

To establish the proposed hybrid EHO technique, its performance is analyzed for single objective optimization problem. A single objective DG allocation problem of 33-bus distribution system is solved for power loss minimization, as suggested in $[6,22]$. The obtained simulation results are compared with different variants of EHO available in existing literature and presented in Table 1. The comparison is obtained for 50 independent trials with same population size and maximum iteration of 50 and 100 respectively, as considered in [6]. The table summarizes best fitness, worst fitness, mean fitness, standard deviation and CPU time. The comparison shows that the proposed hybrid EHO-PSO method provides promising results as compared to available variants of EHO. Although, the computation time of proposed approach is more than the existing variants. The possible cause of high computation may be the use of different load flow calculation methods and insertion of extra steps from PSO. Table 1 validates that the proposed EHO-PSO approach is providing compromising solutions for DGs integration problems. However, it is not providing adequate information of individual improvements suggested in this work. Therefore, effect of individual improvement suggested in the algorithm is further analyzed.

Table 1. Performance comparison of EHO, IEHO and hybrid EHO-PSO for 50 independent trails

\begin{tabular}{|c|c|c|c|c|c|}
\hline Method & $\begin{array}{l}\text { Best } \\
\text { Fitness }\end{array}$ & $\begin{array}{l}\text { Worst } \\
\text { Fitness }\end{array}$ & $\begin{array}{l}\text { Mean } \\
\text { Fitness }\end{array}$ & $\begin{array}{l}\text { Standard } \\
\text { Deviation }\end{array}$ & $\begin{array}{l}\mathrm{CPU} \\
\text { time (s) }\end{array}$ \\
\hline $\mathrm{EHO}[\mathrm{c}$ & 0.0758 & 0.0891 & 0.0807 & 0.003 & 3.19 \\
\hline IEHO [6] & 0.0715 & 0.0806 & 0.0759 & 0.002 & 3.31 \\
\hline EHO-PSO & 0.0714 & 0.0749 & 0.0730 & 0.0007 & 7.01 \\
\hline
\end{tabular}




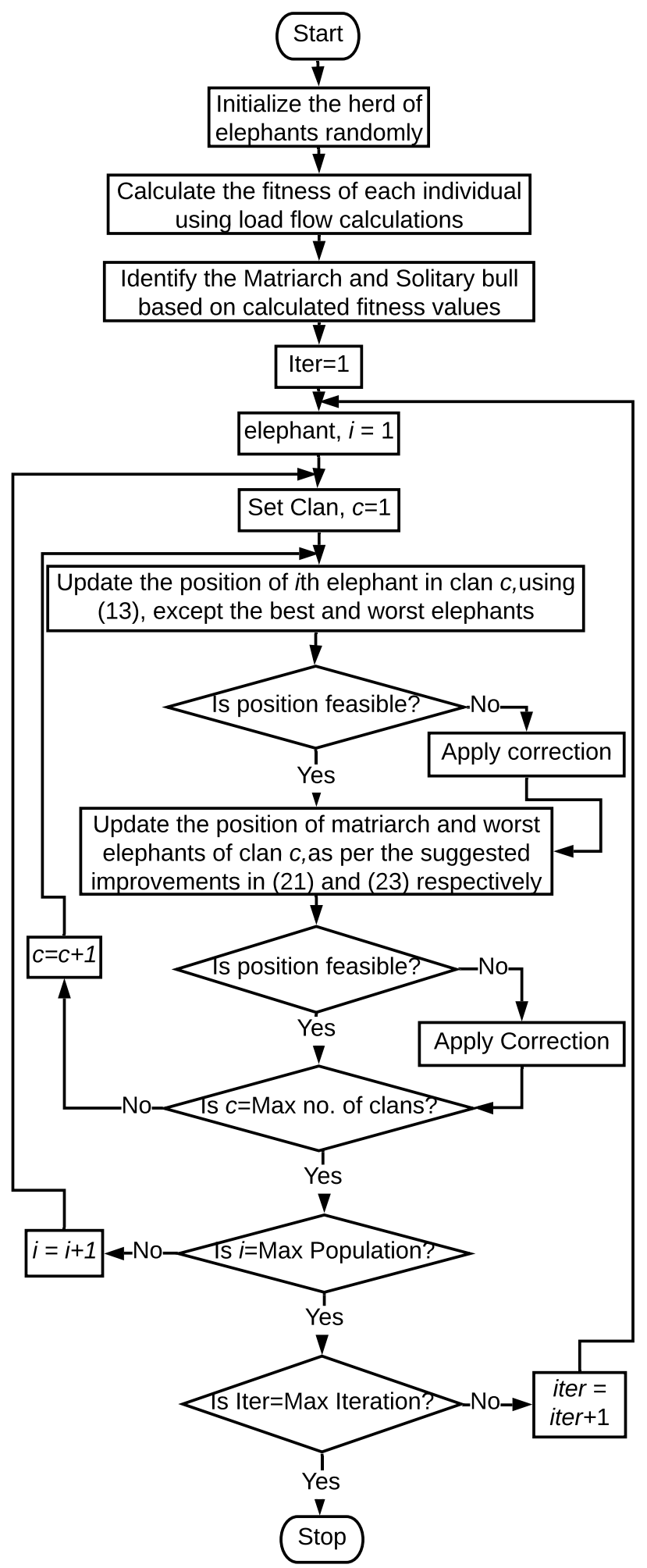

Figure 3. Flowchart of the proposed hybrid EHO-PSO optimization 
Figure 4 shows the box plots of solutions obtained by standard variants of EHO and PSO along with the proposed hybrid EHO-PSO for 50 independent trials. It can be observed from the figure that median of PSO is less than the median of EHO but the solutions obtained by PSO are more scattered. On the other hand, the proposed hybrid EHO-PSO approach provides more evenly distributed solutions with lowest median, as compared to basic variants of EHO and PSO. Similarly, Figure 5 shows the convergence characteristics of standard EHO, standard PSO, EHO with correction-I only, EHO with correction-II only, and EHO with both the corrections (hybrid EHO-PSO). It has observed that standard variants of EHO and PSO are unable to search the global region for DG allocation problems. The suggested improvement-I enhances the gradual searching ability of the standard EHO. Similarly, the suggested improvement-II guides herd to avoid local trappings by controlling the elephants' velocity. By combining these two improvements, the global searching ability of standard EHO has significantly improved.

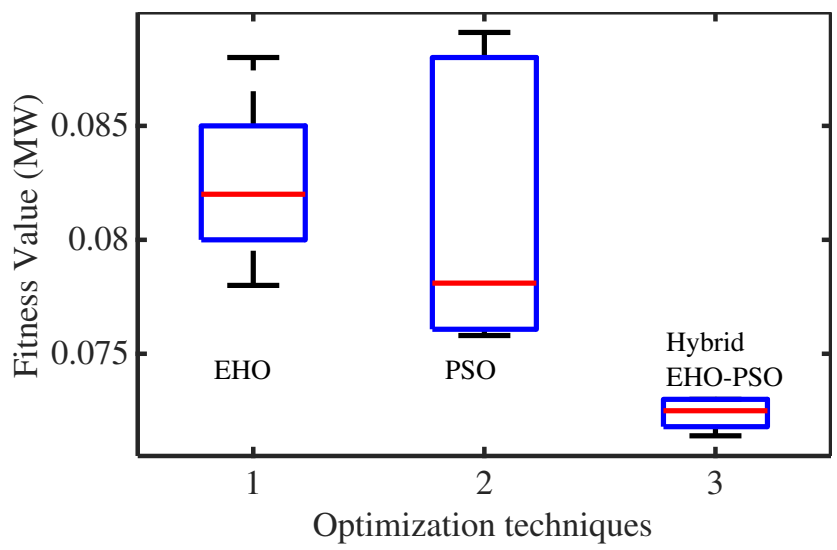

Figure 4. Box plots of solutions obtained by PSO, EHO and hybrid EHO-PSO

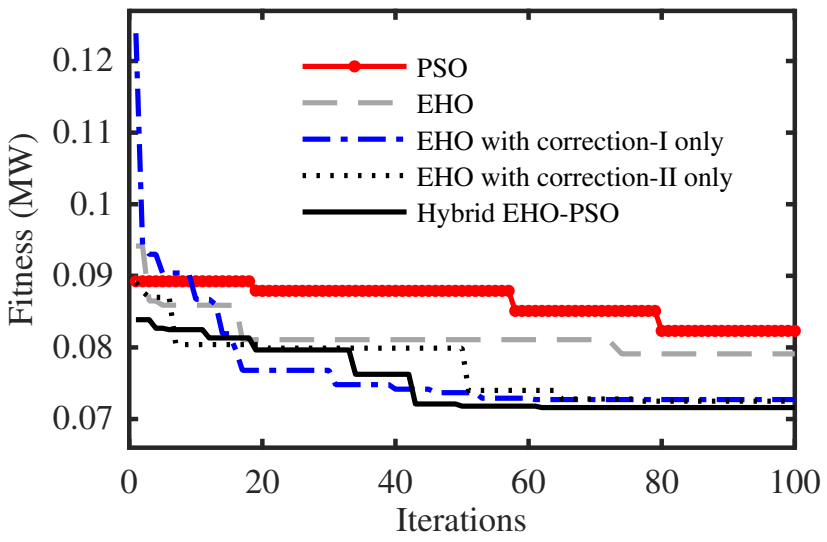

Figure 5. Comparison of convergence characteristics of PSO, EHO, improved EHOs and hybrid EHO-PSO

Now, the potential of proposed hybrid EHO-PSO method is examined for multi-objective DG integration problem of distribution systems. In following sections, the multiobjective DG allocation problem presented in Section 2 is 
solved for three standard test distribution systems and obtained simulation results are compared with some of the well-known optimization techniques in literature.

\subsection{Proposed Multi-objective DG Integration Problem}

In this section, the proposed hybrid EHO-PSO optimization method is now adopted to solve the multi-objective DG integration problem of distribution systems. According to IEEE standard 1547, DG with a capacity less than 10 MW may not be allowed to take part in network regulation at a node of common coupling and should operate at pre-defined power factor [3]. Therefore, the DGs are allowed to operate at pre-defined power factors and considered as negative loads, as also suggested in $[3,5,22]$. In order to compare the simulation results with existing solution available in literature, the values of coefficients $k_{1}$ and $k_{2}$ are referred from $[4,5]$. Furthermore, Newton-Raphson load flow method is adopted to perform the power flow calculations in distribution systems. The optimization parameters used in the simulation are: $\alpha=0.5$ and $\beta=0.1$, as suggested in [27]. The population size and maximum number of iterations considered for system-I and II are 50 and 100 respectively. The problem is formulated in MATLAB environment and simulated on a Inter(R) Core(TM) i5-6200U CPU@2.30GHz processor with 8 GB RAM. The simulated test systems are individually discussed in following sections.

\subsubsection{Study system-I (33-bus test distribution system) In this section, the proposed multi-objective DG allocation} problem is formulated for benchmark 33-bus radial distribution system [29] and solved by using proposed hybrid EHO-PSO method. This system is a $12.66 \mathrm{kV}$ distribution system with the nominal real and reactive power demands of 3.715 MW and 2.300 MVAr respectively. In order to show the effect of DG number and type, three test cases have been investigated for the system, as suggested in literature.

a) 3 DGs operating at unity power factor (UPF)(case-I): In this case, three DGs are assumed to be installed at three different nodes in the system, which are operating at UPF, as suggested in [3-5,30]. The obtained simulation results are compared with existing optimal solutions available in literature and then presented in Table 2 . The table presents optimal DG sites and sizes, values of objective functions, DGs penetration, percentage loss reduction and CPU time. It is observed that the results obtained by the proposed method provides most compromising solution for multi-objective DG integration problem of distribution systems as compared to the methods available in existing literature such as GA, PSO, GA/PSO, TLBO, QOTLBO, Taguchi method (TM), and multi-objective Taguchi approach (MOTA). The performance of active distribution system is significantly improved by proposed method in terms of power loss reduction, node voltage deviation reduction and maximum VSI. The VSI is used to measure the level of line stability in distribution system over variable loading condition. The higher value of VSI indicates the robustness of a line against voltage stability problems. The simulation time of hybrid EHO-PSO method is higher than the MOTA. It is due to the insertion of extra PSO steps to modified EHO. Moreover, the DGs sites in MOTA were selected by using an assisting roulette wheel selection approach to reduce the simulation time significantly.

b) 4 DGs operating at unity power factor (UPF)(case-II): 4 DGs operating at UPF are adopted to install on four different nodes in the system $[3,15,22]$. A comparison of simulation results obtained by different optimization 
Table 2. Comparison of simulation obtained for 33-bus test distribution system (Study System-I)

\begin{tabular}{|c|c|c|c|c|c|c|c|c|}
\hline 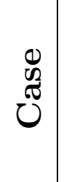 & $\begin{array}{l}\text { Optimization } \\
\text { method }\end{array}$ & $\begin{array}{l}\text { Optimal sites (sizes in } \\
M W \text { ) of DGs }\end{array}$ & $\begin{array}{l}f_{1} \\
(\mathrm{MW})\end{array}$ & $f_{2}$ & $f_{3}$ & $\begin{array}{l}\% \text { DG } \\
\text { pene- } \\
\text { tration }\end{array}$ & $\begin{array}{l}\text { \% Loss } \\
\text { reduc- } \\
\text { tion }\end{array}$ & $\begin{array}{l}\text { CPU } \\
\text { time } \\
\text { (s) }\end{array}$ \\
\hline \multicolumn{2}{|c|}{ Base Case } & & 0.2027 & 0.1171 & 0.6988 & - & - & - \\
\hline \multirow{9}{*}{$\mathrm{I}$} & GA [5] & 11(1.500), 29(0.423), 30(1.071) & 0.1063 & 0.0407 & 0.9490 & 42.86 & 47.56 & - \\
\hline & PSO $[5]$ & 08(1.177), 13(0.982), 32(0.830) & 0.1053 & 0.0335 & 0.9256 & 42.76 & 48.05 & - \\
\hline & GA/PSO [5] & $11(0.925), 16(0.863), 32(1.200)$ & 0.1034 & 0.0124 & 0.9508 & 42.74 & 48.99 & - \\
\hline & TLBO $[18]$ & $09(0.885), 18(0.895) 31(1.196)$ & 0.1040 & 0.0295 & 0.9547 & 42.57 & 48.69 & - \\
\hline & TLBO $[4]$ & 12(1.183), 28(1.191), 30(1.186) & 0.1247 & 0.0011 & 0.9503 & 50.93 & 38.48 & 12.63 \\
\hline & QOTLBO [4] & 13(1.083), 26(1.188), 30(1.199) & 0.1034 & 0.0011 & 0.9530 & 49.64 & 48.99 & 12.55 \\
\hline & TM $[30]$ & $15(0.719), 26(0.719), 33(1.439)$ & 0.1023 & 0.0040 & 0.9371 & 41.16 & 49.53 & 7.92 \\
\hline & MOTA [3] & $30(1.340), 07(0.980), 14(0.960)$ & 0.0963 & 0.0014 & 0.9551 & 46.92 & 52.49 & 0.30 \\
\hline & EHO-PSO & $14(1.148), 24(1.188), 30(1.621)$ & 0.0948 & 0.0008 & 0.9639 & 56.61 & 53.23 & 7.35 \\
\hline \multirow{7}{*}{ II } & GA $[15]$ & $\begin{array}{l}06(0.643), 13(0.857), 24(0.857), \\
30(0.738)\end{array}$ & 0.0701 & 0.0115 & 0.8776 & 44.28 & 65.42 & - \\
\hline & PSO [15] & $06(0.830), 15(0.833)$ & 0.0713 & 0.0109 & 0.8776 & 40.80 & 64.82 & - \\
\hline & & $25(0.541), 31(0.648)$ & & & & & & \\
\hline & GA/PSO [15] & $\begin{array}{l}14(0.663), 24(1.023), 26(0.867), \\
32(0.664)\end{array}$ & 0.0682 & 0.0130 & 0.8903 & 46.02 & 66.35 & - \\
\hline & IMOHS [22] & $\begin{array}{l}06(0.937), 14(0.667), 24(1.012), \\
31(0.731)\end{array}$ & 0.0678 & 0.0111 & 0.8891 & 47.88 & 66.55 & - \\
\hline & MOTA [3] & $\begin{array}{l}24(0.960), 07(1.00), 32(0.680), \\
15(0.560)\end{array}$ & 0.0663 & 0.0111 & 0.8907 & 45.77 & 67.29 & 0.33 \\
\hline & EHO-PSO & $\begin{array}{l}07(0.810), 14(0.625), 24(1.013), \\
32(0.740)\end{array}$ & 0.0663 & 0.0109 & 0.8932 & 45.60 & 67.29 & 11.01 \\
\hline \multirow{7}{*}{ III } & IWO [8] & $\begin{array}{l}14(0.517), \\
32(1.0842)\end{array}$ & 0.3705 & - & - & 24.53 & 81.64 & - \\
\hline & Analytical [31] & $06(1.810), 25(0.840), 31(0.930)$ & 0.0261 & 0.0086 & 0.8678 & 51.22 & 87.12 & - \\
\hline & LSFSA $[7]$ & 06(1.383), 18(0.552), 30(1.063) & 0.0267 & - & - & 42.92 & 86.83 & - \\
\hline & IA [32] & 06(1.059), 14(0.741), 30(1.059) & 0.0231 & - & - & 40.90 & 88.60 & - \\
\hline & TM $[3]$ & $16(0.705), 27(0.705), 30(1.14)$ & 0.0274 & 0.0010 & 0.9545 & 36.38 & 86.48 & - \\
\hline & MOTA $[3]$ & $14(0.880), 25(0.920), 30(1.560)$ & 0.0157 & 0.0004 & 0.9760 & 48.07 & 92.25 & 0.30 \\
\hline & EHO-PSO & $14(0.842), 24(1.281), 30(1.456)$ & 0.0146 & 0.0004 & 0.9740 & 51.20 & 92.79 & 11.29 \\
\hline
\end{tabular}


methods are presented in Table 2. It shows that the proposed approach provides most competitive results with reference to GA, PSO, GA/PSO, IMOHS and MOTA at adequate DG penetration. Similar to previous case, most compromising sites and sizes of UPF DGs are provided by the proposed method. It has been analyzed that increasing number of DGs reduces the system power loss but not able to improve the system performance in terms of node voltage profile and stability.

c) 3 DGs operating at 0.85 lagging power factor (LPF) (Case-III): It has been observed that a DG operating at UPF is not providing reactive power support to the system. Therefore, 3 DGs operating at LPF are considered to install at three different nodes $[3,7,8,31,32]$. The simulation results obtained by proposed hybrid EHOPSO are also validated for this case with well-known optimization techniques such as LSFSA, IA, TM and MOTA, as shown in Table 2. The proposed method provides highest loss reduction with identical node voltage deviation to MOTA. However, the value of VSI provided by the proposed method is slightly less than that of MOTA [3]. It may be concluded that proposed method provides vulnerable solution for utilities and consumers. It has been observed that a DG operating at $0.85 \mathrm{LPF}$ is significantly improve the system performance in contrast to a DG operating at $\mathrm{UPF}$, as it provides reactive power support to the system.

The node voltage profiles of all above investigated cases are presented in Figure 6 . The figure shows that the optimal deployment of DGs has improved the system node voltage profile significantly, as compared to base case condition. Furthermore, it can be analyzed that the consideration of reactive power support uniformly improves the system node voltage profile with respect to UPF DGs. From case-I and II, it has been validated that the increasing number of DGs reduces the DG penetration in the system. Besides, the consideration of reactive power support from DGs also reduces the penetration level, for the same number of DGs.

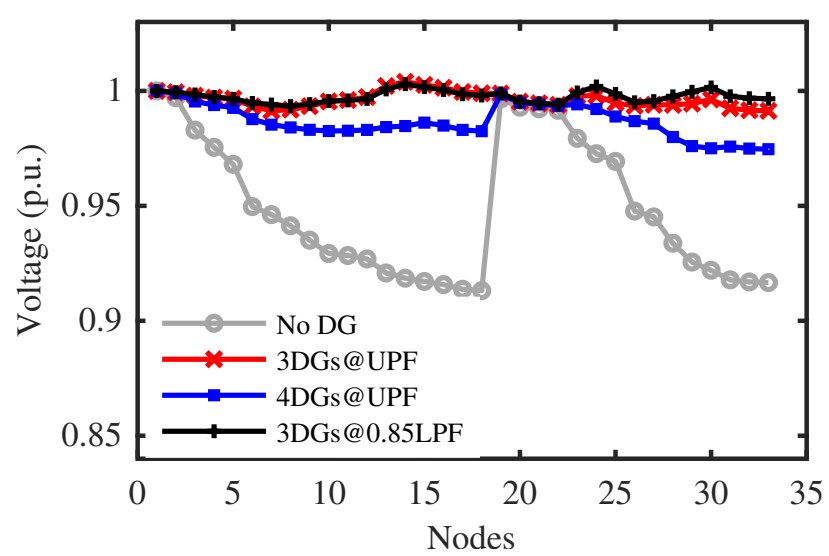

Figure 6. Node voltage profiles of benchmark 33-bus radial distribution system

7.2.2 Study system -II (69-bus test distribution system) The proposed multi-objective DG allocation problem is also solved for benchmark 69-bus [33] radial system to demonstrate the performance of proposed method on mediumscale distribution systems. This is a $12.66 \mathrm{kV}$ power network with total load demand of 3.80 MW, 2.690 MVAr. The 
real and reactive power losses calculated in base case condition are 224.7 KW and 102.13 KVAr respectively. Here, two cases have been investigated for this system using 3 DGs operating at UPF and $0.85 \mathrm{LPF}$, as presented below.

a) 3 DGs operating at UPF (case-I): In this case, 3 DGs are considered to deploy at 3 different nodes, the

Table 3. Comparison of simulation obtained for 69-bus test distribution system (Study System-II)

\begin{tabular}{|c|c|c|c|c|c|c|c|c|}
\hline $\begin{array}{l}0 \\
\text { D. } \\
\text { Uీ }\end{array}$ & $\begin{array}{l}\text { Optimization } \\
\text { method }\end{array}$ & $\begin{array}{l}\text { Optimal sites (sizes in } \\
M W \text { ) of DGs }\end{array}$ & $\begin{array}{l}f_{1} \\
(\mathrm{MW})\end{array}$ & $f_{2}$ & $f_{3}$ & $\begin{array}{l}\% \text { DG } \\
\text { pene- } \\
\text { tration }\end{array}$ & $\begin{array}{l}\% \text { Loss } \\
\text { reduc- } \\
\text { tion }\end{array}$ & $\begin{array}{l}\text { CPU } \\
\text { time } \\
(\mathrm{s})\end{array}$ \\
\hline \multicolumn{3}{|c|}{ Base Case } & 0.2247 & 0.0993 & 0.6870 & - & - & \\
\hline \multirow{6}{*}{ I } & GA $[5]$ & $21(0.929), 62(1.075), 64(0.984)$ & 0.0890 & 0.0012 & 0.9705 & 40.11 & 60.39 & - \\
\hline & PSO [5] & $17(0.992), 61(1.199), 63(0.795)$ & 0.0832 & 0.0049 & 0.9676 & 40.09 & 62.97 & - \\
\hline & $\mathrm{GA} / \mathrm{PSO}[5]$ & $21(0.911), 61(1.192), 63(0.885)$ & 0.0811 & 0.0031 & 0.9768 & 40.11 & 63.90 & - \\
\hline & TLBO $[4]$ & 13(1.013), 61(0.990), 62(1.160) & 0.0821 & 0.0008 & 0.9745 & 42.46 & 63.46 & - \\
\hline & QOTLBO [4] & $15(0.811), 61(1.470), 63(1.002)$ & 0.0805 & 0.0007 & 0.9769 & 44.07 & 64.17 & - \\
\hline & EHO-PSO & $09(0.876), 20(0.604), 61(1.994)$ & 0.0766 & 0.0005 & 0.9713 & 46.64 & 65.91 & 13.09 \\
\hline \multirow{3}{*}{ II } & LSFSA [7] & $18(0.549), 60(1.195), 65(0.312)$ & 0.1626 & - & - & 27.60 & 92.77 & - \\
\hline & IWO $[8]$ & $27(0.370), 61(1.090), 65(0.315)$ & 0.1364 & - & - & 23.83 & 93.92 & - \\
\hline & EHO-PSO & $16(0.665), 08(0.874), 61(1.896)$ & 0.0071 & 0.0002 & 0.9815 & 46.11 & 96.84 & 13.45 \\
\hline
\end{tabular}

DGs are assumed to be operated at UPF $[4,5]$. The simulation results obtained by proposed hybrid EHO-PSO are compared with that of the similar available in literature and presented in Table 3. It has been observed that the proposed approach provides most competitive optimal solution, as compared to other optimization methods such as GA, PSO, GA/PSO, TLBO and QOTLBO. In newly proposed solution, the values of two objective functions are the foremost among all compared techniques. The value of VSI is slightly lesser than other solutions available however the proposed approach provides higher loss reduction which is the most desired objective because of monetary aspects.

b) 3 DGs operating at $0.85 \mathrm{LPF}$ (Case-II): In order to provide reactive power support to the system, 3 DGs operating at $0.85 \mathrm{LPF}$ are assumed to install at three different sites. The optimal sites and sizes of these DGs are summarized in Table 3. The comparison of obtained solution is not possible due to non-availability of results for this case. In order to demonstrate the supremacy of proposed method, the results are compared with $[7,8]$ single objective DG integration problems. Despite of solving multi-objective DG integration problem, the proposed method provides higher loss reduction while improving the system performance appreciably in terms of loss reduction, voltage profile and stability improvement. The voltage profiles of the system for all simulated cases are presented in Figure 7 . It shows that the voltage profile of this system has consistently ameliorated at all nodes for both the cases. 


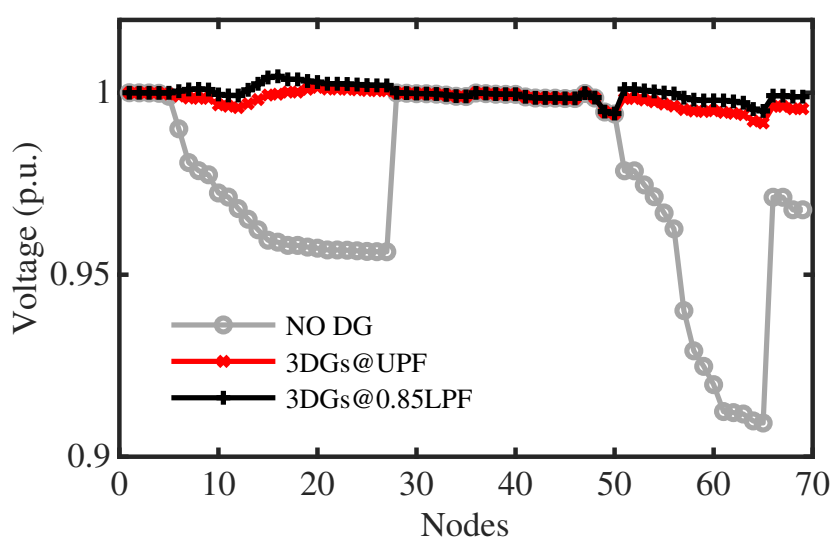

Figure 7. Node voltage profiles of benchmark 69-bus radial distribution system

\subsubsection{Study System -III (118-bus test distribution system) After validation of the proposed hybrid EHO-PSO} method on small and medium size test distribution systems, the effectiveness of proposed technique is implemented on a large benchmark 118-bus radial distributed system [34]. This is a $11 \mathrm{kV}$ large distribution system with total real and reactive power demands of 22.71 MW and 17.04 MVAr respectively. For this system, two cases have been investigated by considering UPF and LPF based DGs.

a) 7 DGs operating at UPF (case-1): In this case, 7 DGs are considered for the placement which are operated at UPF. Optimal nodes and sizes obtained for these DGs using hybrid EHO-PSO method are presented in Table 4. The obtained results are compared with the same available in existing literature $[3,4,7]$. The table shows that the proposed approach provides highest power loss reduction as compared to other techniques, even at lesser DG penetration. However, the value of voltage deviation is slightly more than that of QOTLBO. The value of system VSI is better than other optimization methods. As per the utility perspective, the solution obtained by the suggested technique may be more preferable as it provides high power loss reduction along with adequate values of voltage deviation and VSI values as compared to other methods.

b) 7 DGs operating at 0.85 LPF (case-2): Similar to other systems, it has been concluded that LPF DGs improve the system efficiency significantly in contrast to UPF DGs. Therefore, 7 DGs operating at 0.85 LPF are considered to install at seven different sites in the system. The obtain results are validated with other available techniques in the literature $[3,7]$ and summarized in Table 4 . From the table, it can be noticed that the proposed approach produces superior solution for DG allocation problems in large-scale distribution networks, even at very less DG penetration. The power loss reduction, node voltage profile, and VSI are significantly improved as compared to other optimization techniques. The voltage profiles of the system for both the cases are shown in Fig. 8. It shows that the voltage profile of active distribution system is uniformly improved across all nodes.

The proposed case studies validate the suggested improvement and insertion of PSO operator to EHO. The hybridization of these techniques provides a powerful optimization technique which is found to be very effective as compared to some of the well-known optimization methods such as GA, PSO, TLBO, QOTLBO, TM, MOTA, IMOHS, IWO, LSFSA, etc. The inclusion of mode operator helps the leader elephant to update its position while 
Table 4. Comparison of simulation obtained for 118-bus test distribution system (Study System-III)

\begin{tabular}{|c|c|c|c|c|c|c|c|c|}
\hline 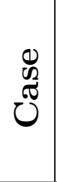 & $\begin{array}{l}\text { Optimization } \\
\text { method }\end{array}$ & $\begin{array}{l}\text { Optimal sites (sizes in MW) of } \\
\text { DGs }\end{array}$ & $\begin{array}{l}f_{1} \\
(\mathrm{MW})\end{array}$ & $f_{2}$ & $f_{3}$ & $\begin{array}{l}\% \text { DG } \\
\text { pene- } \\
\text { tration }\end{array}$ & $\begin{array}{l}\% \text { Loss } \\
\text { reduc- } \\
\text { tion }\end{array}$ & $\begin{array}{l}\text { CPU } \\
\text { time } \\
(\mathrm{s})\end{array}$ \\
\hline \multicolumn{2}{|c|}{ Base Case } & & 1.2981 & 0.3576 & 0.5703 & - & - & - \\
\hline \multirow{5}{*}{ I } & $\mathrm{SA}[7]$ & $\begin{array}{l}036(7.467), 048(0.711), 056(3.673), \\
075(2.824), 088(2.297), 103(5.080), \\
116(0.461)\end{array}$ & 0.9001 & - & - & 49.57 & 30.66 & 25.30 \\
\hline & TLBO [4] & $\begin{array}{l}035(3.246), 048(2.886), 065(2.430), \\
072(3.305), 086(1.991), 099(1.604), \\
111(3.598)\end{array}$ & 0.7058 & 0.0327 & 0.8548 & 41.97 & 45.63 & 20.91 \\
\hline & QOTLBO [4] & $\begin{array}{l}043(1.588), 049(3.845), 054(0.985), \\
074(3.190), 080(3.163), 094(1.952), \\
0111(3.601)\end{array}$ & 0.6776 & 0.0233 & 0.8794 & 40.35 & 47.80 & 20.85 \\
\hline & MOTA [3] & $\begin{array}{l}033(5.640), 042(1.680), 060(4.380), \\
072(0.960), 078(3.420), 092(2.100), \\
108(3.180)\end{array}$ & 0.6182 & 0.0305 & 0.8797 & 47.03 & 52.37 & - \\
\hline & EHO-PSO & $\begin{array}{l}018(3.852), 042(1.716), 050(3.679), \\
074(2.708), 079(2.456), 091(1.875), \\
109(3.259)\end{array}$ & 0.5597 & 0.0348 & 0.8910 & 38.90 & 56.88 & 33.15 \\
\hline \multirow{3}{*}{ II } & $\mathrm{SA}[7]$ & $\begin{array}{l}036(7.056), 048(2.515), 056(4.980) \\
075(3.181), 088(0.723), 103(6.161) \\
116(0.586)\end{array}$ & 0.6348 & - & - & 55.49 & 51.10 & 25.30 \\
\hline & MOTA [3] & $\begin{array}{l}033(5.640), 042(1.680), 060(4.380), \\
072(0.960), 078(3.420), 092(2.100), \\
108(3.180)\end{array}$ & 0.2329 & 0.0138 & 0.8825 & 47.03 & 82.06 & - \\
\hline & EHO-PSO & $\begin{array}{l}020(2.290), 040(2.680), 050(3.591), \\
073(3.000), 080(2.709), 091(1.865), \\
110(3.578)\end{array}$ & 0.1446 & 0.0077 & 0.9128 & 37.44 & 88.86 & 34.23 \\
\hline
\end{tabular}




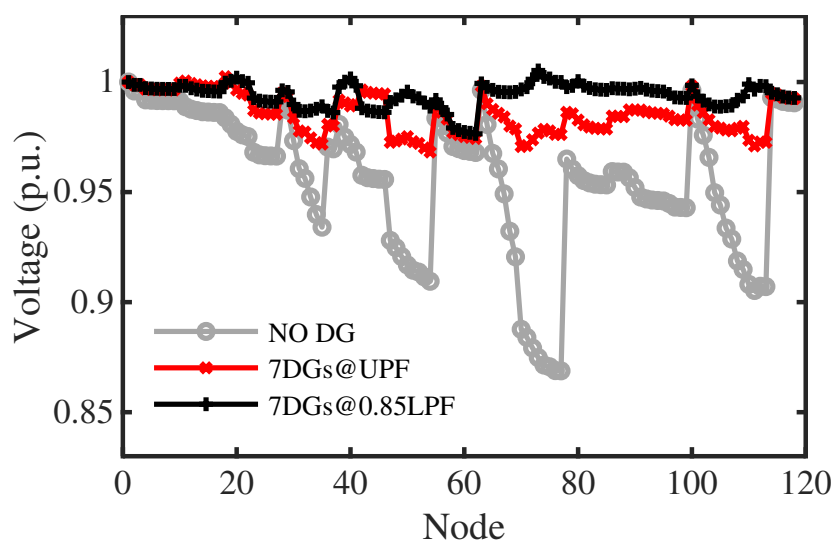

Figure 8. Node voltage profiles of benchmark 118-bus radial distribution system

considering the positions of majority elephants in that clan. It is well suited for DG allocation problems since every system has some sets of nodes which are having optimal solution and keeps on repeating during the iterations. The method provides inspiring solution for utilities to meliorate the system performance in terms of power loss reduction, voltage profile and stability improvement as compared to other methods.

\section{Conclusion}

In this article, an effective hybrid EHO-PSO method is proposed to solve the DG allocation problem of distribution systems. In the proposed hybridization, an improvement is suggested in standard EHO. Furthermore, a powerful trait of PSO, i.e. velocity operator, is introduced for worst elephants in EHO. The inclusion of a velocity operator in EHO helps the worst elephants to remain in touch with their respective family groups. The suggested modifications have been validated for single objective DG allocation problems.

After validation, the proposed method is adopted to solve multi-objective DG placement problems of three distribution networks. A multi-objective DG allocation problem is formulated to ameliorate the performance of distribution systems. The objective functions considered are power loss minimization, voltage deviation minimization, and voltage stability improvement. The considered objectives are esteemed measures for utilities and customers since these exhibit various techno-economic performance of distribution systems. The acquired simulation results are providing better choices to utility engineers for the smooth operations of active distribution networks. Simulation results reveal that the proposed approach is significantly improved the performance of small to large-scale distribution systems with reference to many of the existing optimization methods.

We believe that the proposed hybrid optimization technique may be highly applicable to solve the various single and multi-objective optimization problems encountered in daily industry practices.

\section{Acknowledgment}

This project has received funding from the European Union's Horizon 2020 research and innovation program under the Marie Sklodowska-Curie grant agreement No 713694. 


\section{References}

[1] W. El-Khattam and M. M. A. Salama, "Distribution system planning using distributed generation," in $C C E C E$ 2003 - Canadian Conference on Electrical and Computer Engineering. Toward a Caring and Humane Technology (Cat. No.03CH37436), vol. 1, May 2003, pp. 579-582.

[2] P. S. Georgilakis and N. D. Hatziargyriou, "Optimal distributed generation placement in power distribution networks: Models, methods, and future research," IEEE Transactions on Power Systems, vol. 28, no. 3, pp. 3420-3428, Aug 2013.

[3] N. K. Meena, A. Swarnkar, N. Gupta, and K. R. Niazi, "Multi-objective taguchi approach for optimal dg integration in distribution systems," IET Generation, Transmission Distribution, vol. 11, no. 9, pp. 2418-2428, 2017.

[4] S. Sultana and P. K. Roy, "Multi-objective quasi-oppositional teaching learning based optimization for optimal location of distributed generator in radial distribution systems," International Journal of Electrical Power E3 Energy Systems, vol. 63 , pp. $534-545,2014$.

[5] M. Moradi and M. Abedini, "A combination of genetic algorithm and particle swarm optimization for optimal dg location and sizing in distribution systems," International Journal of Electrical Power 6 Energy Systems, vol. 34, no. 1, pp. $66-74,2012$.

[6] N. K. Meena, S. Parashar, A. Swarnkar, N. Gupta, and K. R. Niazi, "Improved elephant herding optimization for multiobjective der accommodation in distribution systems," IEEE Transactions on Industrial Informatics, vol. 14, no. 3, pp. 1029-1039, March 2018.

[7] S. K. Injeti and N. P. Kumar, "A novel approach to identify optimal access point and capacity of multiple dgs in a small, medium and large scale radial distribution systems," International Journal of Electrical Power ES Energy Systems, vol. 45, no. 1, pp. $142-151,2013$.

[8] D. R. Prabha and T. Jayabarathi, "Optimal placement and sizing of multiple distributed generating units in distribution networks by invasive weed optimization algorithm," Ain Shams Engineering Journal, vol. 7, no. 2, pp. 683 - 694, 2016.

[9] N. Kanwar, N. Gupta, K. Niazi, A. Swarnkar, and R. Bansal, "Simultaneous allocation of distributed energy resource using improved particle swarm optimization," Applied Energy, vol. 185, pp. 1684 - 1693, 2017.

[10] H. L. Willis, "Analytical methods and rules of thumb for modeling dg-distribution interaction," in 2000 Power Engineering Society Summer Meeting (Cat. No.00CH37134), vol. 3, 2000, pp. 1643-1644.

[11] D. Q. Hung, N. Mithulananthan, and R. C. Bansal, "Analytical expressions for dg allocation in primary distribution networks," IEEE Transactions on Energy Conversion, vol. 25, no. 3, pp. 814-820, Sept 2010.

[12] W.-S. Tan, M. Y. Hassan, M. S. Majid, and H. A. Rahman, "Optimal distributed renewable generation planning: A review of different approaches," Renewable and Sustainable Energy Reviews, vol. 18, pp. 626 - 645, 2013.

[13] N. K. Meena, A. Swarnkar, J. Yang, N. Gupta, and K. R. Niazi, "Modified taguchi-based approach for optimal distributed generation mix in distribution networks," IEEE Access, vol. 7, pp. 135 689-135 702, 2019.

[14] A. Muqbel, A. H. Elsayed, M. A. Abido, A.-A. Mantawy, A. T. Al-Awami, and M. El-Hawary, "Optimal sizing and location of solar capacity in an electrical network using lightning search algorithm," Electric Power Components and Systems, vol. 47, no. 14-15, pp. 1247-1260, 2019.

[15] M. H. Moradi and M. Abedinie, "A combination of genetic algorithm and particle swarm optimization for optimal dg location and sizing in distribution systems," in 2010 Conference Proceedings IPEC, Oct 2010, pp. 858-862. 
[16] J. Kennedy and R. Eberhart, "Particle swarm optimization," in Neural Networks, 1995. Proceedings., IEEE International Conference on, vol. 4, Nov 1995, pp. 1942-1948.

[17] P. Kumar, I. Ali, M. S. Thomas, and S. Singh, "A coordinated framework of dg allocation and operating strategy in distribution system for configuration management under varying loading patterns," Electric Power Components and Systems, vol. 0, no. 0, pp. 1-18, 2020.

[18] B. Mohanty and S. Tripathy, "A teaching learning based optimization technique for optimal location and size of dg in distribution network," Journal of Electrical Systems and Information Technology, vol. 3, no. 1, pp. 33 - 44, 2016.

[19] A. A. A. El-Ela, R. A. El-Sehiemy, and A. S. Abbas, "Optimal placement and sizing of distributed generation and capacitor banks in distribution systems using water cycle algorithm," IEEE Systems Journal, vol. 12, no. 4, pp. 3629 $-3636,2018$.

[20] M. Tolba, H. Rezk, A. Diab, and M. Al-Dhaifallah, "A novel robust methodology based salp swarm algorithm for allocation and capacity of renewable distributed generators on distribution grids," Energies, vol. 11, no. 10, p. $2556,2018$.

[21] N. Kanwar, N. Gupta, K. R. Niazi, and A. Swarnkar, "Optimal allocation of dgs and reconfiguration of radial distribution systems using an intelligent search-based tlbo," Electric Power Components and Systems, vol. 45, no. 5, pp. 476-490, 2017.

[22] K. Nekooei, M. M. Farsangi, H. Nezamabadi-Pour, and K. Y. Lee, "An improved multi-objective harmony search for optimal placement of dgs in distribution systems," IEEE Transactions on Smart Grid, vol. 4, no. 1, pp. 557-567, March 2013.

[23] J. Siahbalaee, N. Rezanejad, and G. B. Gharehpetian, "Reconfiguration and dg sizing and placement using improved shuffled frog leaping algorithm," Electric Power Components and Systems, vol. 47, no. 16-17, pp. 1475-1488, 2019.

[24] N. MEENA, A. Swarnkar, N. Gupta, and K. Niazi, "Optimal integration of ders in coordination with existing voltage regulators in distribution networks," IET Generation, Transmission ES Distribution, vol. 12, no. 11, 2018.

[25] P. Singh, S. Bishnoi, and N. K. Meena, "Moth search optimization for optimal ders integration in conjunction to oltc tap operations in distribution systems," IEEE Systems Journal, vol. 14, no. 1, pp. 880-888, 2020.

[26] M. A. Tolba, A. A. Zaki Diab, A. S. Vanin, V. N. Tulsky, and A. Y. Abdelaziz, "Integration of renewable distributed generation in distribution networks including a practical case study based on a hybrid psogsa optimization algorithm," Electric Power Components and Systems, vol. 46, no. 19-20, pp. 2103-2116, 2018.

[27] G. G. Wang, S. Deb, and L. d. S. Coelho, "Elephant herding optimization," in 2015 3rd International Symposium on Computational and Business Intelligence (ISCBI), Dec 2015, pp. 1-5.

[28] M. Chakravorty and D. Das, "Voltage stability analysis of radial distribution networks," International Journal of Electrical Power \& Energy Systems, vol. 23, no. 2, pp. 129-135, 2001.

[29] M. E. Baran and F. F. Wu, "Network reconfiguration in distribution systems for loss reduction and load balancing," IEEE Transactions on Power Delivery, vol. 4, no. 2, pp. 1401-1407, Apr 1989.

[30] N. K. Meena, A. Swarnkar, N. Gupta, and K. R. Niazi, "A taguchi-based approach for optimal placement of distributed generations for power loss minimization in distribution system," in 2015 IEEE Power Energy Society General Meeting, July 2015, pp. 1-5.

[31] S. N. G. Naik, D. K. Khatod, and M. P. Sharma, "Analytical approach for optimal siting and sizing of distributed generation in radial distribution networks," IET Generation, Transmission Distribution, vol. 9, no. 3, pp. 209-220, 2015 . 
[32] D. Q. Hung and N. Mithulananthan, "Multiple distributed generator placement in primary distribution networks for loss reduction," IEEE Transactions on Industrial Electronics, vol. 60, no. 4, pp. 1700-1708, April 2013.

[33] M. E. Baran and F. F. Wu, "Optimal capacitor placement on radial distribution systems," IEEE Transactions on Power Delivery, vol. 4, no. 1, pp. 725-734, Jan 1989.

[34] D. Zhang, Z. Fu, and L. Zhang, "An improved ts algorithm for loss-minimum reconfiguration in large-scale distribution systems," Electric Power Systems Research, vol. 77, no. 5, pp. 685 - 694, 2007. 